\title{
Spot urine protein to creatinine ratio
}

\author{
Guido Filler ${ }^{1,2,3,4} \mathbb{C} \cdot$ Shih-Han Susan Huang ${ }^{1,3}$
}

Received: 16 January 2017 / Accepted: 18 January 2017 / Published online: 14 February 2017

(C) IPNA 2017

\begin{abstract}
In a recent article in Pediatric Nephrology, EM Yang and colleagues (Pediatr Nephrol 2017: doi:10.1007/ s00467-016-3587-6) published a retrospective crosssectional study involving a cohort of 442 children with an mean estimated glomerular filtration rate of $>60 \mathrm{~mL} / \mathrm{min} / 1$. $73 \mathrm{~m}^{2}$. The authors measured 24-h urine protein excretion (24-h UProt) alongside the morning spot urine protein to creatinine ratio (Prot/Cr) in this group of patients. While the Prot/ $\mathrm{Cr}$ may be the only feasible way to routinely estimate the daily protein excretion of a young child, inter-individual variability in childrens' urinary creatinine excretion ( $\mathrm{UCr}$ ) may heavily influence the result. The authors sought to determine which equation was the most accurate in predicting UCr. Not only did they discover that the adult Cockcroft-Gault equation worked best, they also found that multiplying the Prot/Cr by the estimated UCr significantly improved the accuracy of the 24-h UProt estimate. In this editorial we discuss both the strengths and limitations of the study by EM Yang and colleagues. We also highlight the importance of adhering to internationally agreed upon reporting guidelines such as the
\end{abstract}

Guido Filler

guido.filler@1hsc.on.ca

1 Department of Pediatrics, Schulich School of Medicine \& Dentistry, Western University, London, ON, Canada N6A 5W9

2 Department of Pathology and Laboratory Medicine, Schulich School of Medicine \& Dentistry, Western University, London, ON, Canada N5A 5 A5

3 Department of Medicine, Schulich School of Medicine \& Dentistry, Western University, London, ON, Canada N6A 5W9

4 Department of Pediatrics, Children's Hospital, London Health Science Centre, Western University, 800 Commissioners Road East, London, ON, Canada N6A 5W9
STrengthening the Reporting of OBservational studies in Epidemiology (STROBE) statement.

Keywords Proteinuria $\cdot$ Creatinine excretion rate $\cdot 24$-h urine protein $\cdot$ Reporting quality

\section{Introduction}

Determining the degree of proteinuria in a patient is essential in the diagnosis and management of any renal disease. The gold standard for measuring proteinuria is the 24-h urinary protein excretion (24-h UProt) method. However, 24-h urine collection is especially difficult in young children and may not be feasible, and it is notoriously unreliable in older children. Random urine samples are therefore preferred, and the urinary protein to creatinine ratio (UProt/Cr) is widely used to assess a child's protein excretion [1]. Carolyn Abitbol and her group used regression analysis to demonstrate to clinicians that they must determine daily protein excretion using total protein $\left(\mathrm{gm} / \mathrm{m}^{2} /\right.$ day $)=0.63(\mathrm{UProt} / \mathrm{Cr})$ at all levels of proteinuria [2]. Unfortunately, the urinary creatinine concentration and intra- and inter-individual variability in creatinine excretion substantially influence the UProt/Cr. Recently, CY Yang et al. elegantly demonstrated that the "UProt/Cr in samples with low or high specific gravity is more likely to overestimate or underestimate actual daily urine protein amount, respectively, especially in a dilute urine sample with its creatinine below $38.8 \mathrm{mg} / \mathrm{dL}$ or a concentrated sample with its creatinine above $61.5 \mathrm{mg} / \mathrm{dL}$ " [3]. These authors concluded that "...UPCR results should be interpreted with caution in cases that involve dilute urine samples because its overestimation may lead to an erroneous diagnosis of proteinuric renal disease." In view of these findings, we were delighted to come upon the study published by EM Yang et al. in this edition of Pediatric 
Nephrology [4]. The authors carried out a retrospective crosssectional cohort study that included 692 children with an estimated glomerular filtration rate (eGFR) of $>60 \mathrm{~mL} / \mathrm{min} / 1.73 \mathrm{~m}^{2}$. They measured 24-h UProt excretion alongside the morning spot UProt/Cr ratio in this cohort of patients and sought to determine which equation could most accurately predict urinary creatinine excretion (UCr). Not only did they find that the adult CockcroftGault equation worked best, but they demonstrated that multiplying Prot/Cr by the estimated UCr significantly improves the accuracy of the estimated 24-h protein excretion.

\section{Results of the EM Yang et al. study}

The investigation conducted by EM Yang et al. was a retrospective cohort study [4]. The authors used relatively stringent criteria to identify possible errors in the data set and to identify any problems with the timed urine collection, including an assessment of the 24-h UCr excretion. They used the last $10 \mathrm{~mL}$ of the 24-h collection as the first morning void and the appropriate standardized methods to measure UProt and $\mathrm{UCr}$ for the calculation of the urinary protein to creatinine ratio. They employed various formulae to calculate the estimated 24-h UCr, including the well-known Hellerstein formula [5] and the adult Cockcroft-Gault formula [6]. Interestingly, the Cockcroft-Gault formula worked best for estimating daily UCr excretion, even though it cannot be used to estimate GFR in the pediatric population [7]. The group made use of the appropriate statistical methods, such as the Bland-Altman analysis, to determine the agreement between the measured and estimated daily UCr. They also showed that there was a much better agreement between UProt/Cr and the 24-h UProt if the UProt/Cr was multiplied with the estimated UCr; surprisingly, using the Cockcroft-Gault equation yielded the strongest correlation, with a correlation coefficient of $r=$ 0.847 . This approach therefore appears to improve the accuracy of the daily UProt estimate in children with dilute or concentrated urine. The authors should be congratulated for their study since adopting this approach will greatly enhance the accuracy of spot UProt/Cr testing.

\section{Generalizability and future application}

Should we therefore employ this recommendation in future care? The first step to answering this question is to look at the quality of the reporting in the study. The pediatric nephrology community unfortunately does not have a good track record when it comes to reporting randomized controlled clinical trials using the CONsolidated Standards Of Reporting Trials (CONSORT) criteria or including the appropriate figures and checklists [8]. Trial reporting in field of pediatric renal transplantation has been criticized for its poor compliance with the CONSORT criteria. Of particular concern, the reporting of the essential components of the Methods, Results, and Discussion sections has been described as unsatisfactory [9]. The Enhancing the QUAlity and Transparency Of health Research (EQUATOR) statement (http://www.equator-network.org/) provides a one-stop source for standardized reporting guidelines for common study designs. Since the study by EM Yang et al. [4] is a retrospective cross-sectional study, the authors should have employed the Strengthening the Reporting of Observational Studies in Epidemiology (STROBE) Statement guidelines used for observational studies. This guideline can also be used for casecontrol and cohort studies. Many journals have embraced this stringent approach including the British Medical Journal [10], the Lancet [11], and PLOS Medicine [12]. For example, according to the STROBE guidelines, EM Yang et al. [4] should have included the study design in their title. Unfortunately, however, Pediatric Nephrology requires succinct titles that would preclude the use of "A retrospective study..." The authors did include a flow chart [checklist item 13 (c)]. Another guideline that should have been followed but was not includes consistently providing unadjusted estimates and, if applicable, confounder-adjusted estimates and their precision (e.g., 95\% confidence interval)—standard 16 (a); this is one of several examples. Although Pediatric Nephrology has not yet embraced the EQUATOR statement, the publication of a very important study, such as the one by EM Yang et al., [4] should perhaps trigger a discussion about the importance of embracing such standards, especially when striving for a higher impact factor.

One other question that remains to be answered concerns the best approach for validating the proposed formula for correcting the daily protein excretion estimate based on the first morning void. EM Yang et al. [4] used the entire cohort to generate the formula. The authors could have divided their study participants into one cohort of 160 patients to generate the formula and a second cohort composed of the remaining patients to validate the formula, akin to other biomarker discovery studies $[13,14]$. There are five stages for biomarker development, and the later stages focus on epidemiologic concepts within a diagnostic, prognostic, and screening framework to determine if new markers will advance clinical care [15]. Future studies should consider performing both an internal and an external prospective validation of the proposed formula to firmly establish a revised clinical practice and, in this case, to move from the current simple Prot/Cr to the modified approach proposed by the authors.

Nonetheless, EM Yang et al. [4] applied a number of critically rigorous criteria, such as excluding specific samples and carefully applying the appropriate tests to generate a robust and superior approach to calculating 24-h UProt from a spot sample. The limitations of the study are not discussed in detail, and the bias of using the last $10 \mathrm{~mL}$ of the $24-\mathrm{h}$ urine 
collection for the spot sample limits this new approach to the first morning void. Future prospective studies should include children in various demographic situations, clinical status, and social status to improve the validity and generalizability of these findings. One limitation that was identified, i.e., the potential bias owing to the elimination of 224 participants, could have easily been addressed by comparing key demographic features of the eliminated participants and the included participants.

\section{Conclusion}

The study by EM Yang et al. [4] significantly reduces the potential bias and error introduced by spot UProt/Cr ratios in dilute and concentrated urine, and the undersigned recommend implementing the approach put forward by these authors, particularly when 24-h urine collection is not feasible. The surprising result that the Cockcroft-Gault formula performed better than the Hellerstein formula for estimating the 24-h $\mathrm{Cr}$ excretion must be validated in a prospective cohort study across diverse communities. Future studies that use proteinuria as an outcome marker when 24-h urine collections cannot be used should adopt the approach listed by the authors. We fully agree with the conclusion of the authors, which reads: "The use of eU Prot/CR overcomes the difficulties in calculating the amount of 24-h U Prot and is more accurate than U Prot/CR for predicting 24-h U Prot. The authors recommend the use of eU Prot/CR rather than U Prot/CR for estimating 24-h urinary protein excretion."

\section{Compliance with ethical standards}

Conflict of interest The authors declare there are no conflicts.

\section{References}

1. Ginsberg JM, Chang BS, Matarese RA, Garella S (1983) Use of single voided urine samples to estimate quantitative proteinuria. $\mathrm{N}$ Engl J Med 309:1543-1546
2. Abitbol C, Zilleruelo G, Freundlich M, Strauss J (1990) Quantitation of proteinuria with urinary protein/creatinine ratios and random testing with dipsticks in nephrotic children. J Pediatr 116:243-247

3. Yang CY, Chen FA, Chen CF, Liu WS, Shih CJ, Ou SM, Yang WC, Lin CC, Yang AH (2015) Diagnostic accuracy of urine protein/ creatinine ratio is influenced by urine concentration. PLoS One 10:e0137460

4. Yang EM, Yoon BA, Kim SW, Kim CJ (2017) Clinical utility of spot urine protein-to-creatinine ratio modified by estimated daily creatinine excretion in children. Pediatr Nephrol. doi:10.1007/ s00467-016-3587-6

5. Hellerstein S, Simon SD, Berenbom M, Erwin P, Nickell E (2001) Creatinine excretion rates for renal clearance studies. Pediatr Nephrol 16:637-643

6. Cockcroft DW, Gault MH (1976) Prediction of creatinine clearance from serum creatinine. Nephron 16:31-41

7. Filler G, Foster J, Acker A, Lepage N, Akbari A, Ehrich JH (2005) The Cockcroft-Gault formula should not be used in children. Kidney Int 67:2321-2324

8. Schulz KF, Altman DG, Moher D (2010) CONSORT 2010 statement: updated guidelines for reporting parallel group randomised trials. BMJ 340:c332

9. Brooks RJ, Higgins GY, Webster AC (2010) Systematic review of randomized controlled trial quality in pediatric kidney transplantation. Pediatr Nephrol 25:2383-2392

10. von Elm E, Altman DG, Egger M, Pocock SJ, Gotzsche PC, Vandenbroucke JP (2007) Strengthening the Reporting of Observational Studies in Epidemiology (STROBE) statement: guidelines for reporting observational studies. BMJ 335:806-808

11. von Elm E, Altman DG, Egger M, Pocock SJ, Gotzsche PC, Vandenbroucke JP (2007) The Strengthening the Reporting of Observational Studies in Epidemiology (STROBE) statement: guidelines for reporting observational studies. Lancet 370:14531457

12. von Elm E, Altman DG, Egger M, Pocock SJ, Gotzsche PC, Vandenbroucke JP (2007) The Strengthening the Reporting of Observational Studies in Epidemiology (STROBE) statement: guidelines for reporting observational studies. PLoS Med 4:e296

13. Witzel SH, Huang SH, Braam B, Filler G (2015) Estimation of GFR using beta-trace protein in children. Clin J Am Soc Nephrol 10:401-409

14. Benlamri A, Nadarajah R, Yasin A, Lepage N, Sharma AP, Filler G (2010) Development of a beta-trace protein based formula for estimation of glomerular filtration rate. Pediatr Nephrol 25:485-490

15. Ismail OZ, Bhayana V, Kadour M, Lepage N, Gowrishankar M, Filler G (2017) Improving the translation of novel biomarkers to clinical practice: The story of cystatin $\mathrm{C}$ implementation in Canada: A professional practice column. Clin Biochem. doi:10.1016/j. clinbiochem.2017.01.005 